\title{
MTORC1 regulates cardiac function and myocyte survival through 4E-BP1 inhibition in mice
}

\author{
Denghong Zhang, ${ }^{1}$ Riccardo Contu, ${ }^{2}$ Michael V.G. Latronico, ${ }^{2}$ Jian Ling Zhang, ${ }^{1}$ Roberto Rizzi, ${ }^{2,3}$ \\ Daniele Catalucci, ${ }^{2,3}$ Shigeki Miyamoto, ${ }^{4}$ Katherine Huang, ${ }^{4}$ Marcello Ceci, ${ }^{5}$ Yusu Gu, ${ }^{1}$ \\ Nancy D. Dalton, ${ }^{1}$ Kirk L. Peterson, ${ }^{1}$ Kun-Liang Guan, ${ }^{4}$ Joan Heller Brown, ${ }^{4}$ Ju Chen, ${ }^{1}$ \\ Nahum Sonenberg, ${ }^{6}$ and Gianluigi Condorelli ${ }^{1}$
}

\begin{abstract}
${ }^{1}$ Department of Medicine, University of California San Diego, La Jolla, California, USA. ${ }^{2}$ Casa di Cura Multimedica, Istituto di Ricovero e Cura a Carattere Scientifico, Milan, Italy. ${ }^{3}$ Istituto Tecnologie Biomediche, Consiglio Nazionale Ricerche, Segrate, Italy. ${ }^{4}$ Department of Pharmacology, University of California San Diego, La Jolla, California, USA. ${ }^{5}$ Fondazione Parco Biomedico San Raffaele Roma, Rome, Italy. ${ }^{6}$ Department of Biochemistry, McGill University, Montreal, Canada.
\end{abstract}

\begin{abstract}
Mechanistic target of rapamycin (MTOR) plays a critical role in the regulation of cell growth and in the response to energy state changes. Drugs inhibiting MTOR are increasingly used in antineoplastic therapies. Myocardial MTOR activity changes during hypertrophy and heart failure (HF). However, whether MTOR exerts a positive or a negative effect on myocardial function remains to be fully elucidated. Here, we show that ablation of Mtor in the adult mouse myocardium results in a fatal, dilated cardiomyopathy that is characterized by apoptosis, autophagy, altered mitochondrial structure, and accumulation of eukaryotic translation initiation factor 4E-binding protein 1 (4E-BP1). 4E-BP1 is an MTOR-containing multiprotein complex-1 (MTORC1) substrate that inhibits translation initiation. When subjected to pressure overload, Mtor-ablated mice demonstrated an impaired hypertrophic response and accelerated HF progression. When the gene encoding 4E-BP1 was ablated together with Mtor, marked improvements were observed in apoptosis, heart function, and survival. Our results demonstrate a role for the MTORC1 signaling network in the myocardial response to stress. In particular, they highlight the role of 4E-BP1 in regulating cardiomyocyte viability and in HF. Because the effects of reduced MTOR activity were mediated through increased 4E-BP1 inhibitory activity, blunting this mechanism may represent a novel therapeutic strategy for improving cardiac function in clinical HF.
\end{abstract}

\section{Introduction}

Mechanistic target of rapamycin (MTOR) is a key regulator of protein synthesis in the cardiomyocyte $(1,2)$. Increased protein synthesis underpins hypertrophic growth, a salient feature of the heart when it is subjected to stress (3). However, continuous stress leads to inhibition of protein synthesis, decompensated hypertrophy, and heart failure (HF) (4).

MTOR functions as the catalytic subunit of 2 MTOR complexes: MTOR-containing multiprotein complex-1 (MTORC1) and MTORC2. Regulatory associated protein of MTOR, complex 1 (Raptor); proline-rich Akt substrate $40 \mathrm{KDa}$ (PRAS40); and MTOR-associated protein, LST8 homolog (Mlst8) as well as MTOR itself are the main components of MTORC1 (5). This complex mediates temporal control of cell growth through a plethora of biological effects (6). These include control of mRNA translation initiation via the regulation of the cap-binding eukaryotic translation initiation factor 4E (eIF-4E) through phosphorylation of eIF4E-binding proteins (4E-BPs) (7); control of ribosome biogenesis via stimulation of polymerase I and III transcription (8); control of mitochondrial metabolism through peroxisome proliferator-activated receptor, $\gamma$, coactivator $1 \alpha$ and YY1 transcription factor (9); and suppression of autophagy by negatively regulating the association between autophagy-related gene 1 (Atg1) and Atg13 (10).

Conflict of interest: The authors have declared that no conflict of interest exists Citation for this article: J Clin Invest. 2010;120(8):2805-2816. doi:10.1172/JCI43008.
MTORC2 consists of MTOR, rapamycin-insensitive companion of MTOR (Rictor), Mlst8, mammalian stress-activated protein kinase-interacting protein/mitogen-activated protein kinase-associated protein 1 (mSin1/Mapkap1), and proline-rich 5 like (Prr5l) (11). MTORC2 phosphorylates PKC- $\alpha(12,13)$, serum/glucocorticoid-regulated kinase 1 (Sgk1) (14), and Akt at Ser473 (15). It modulates cell survival via Akt and spatial control of cell growth via PKC- $\alpha$ (12). MTORC2, unlike MTORC1, is not directly inhibited by FK506-binding protein 12-rapamycin (FKBP12-rapamycin), but it can be inhibited in a cell-specific manner after prolonged treatment with rapamycin (16).

We reported previously that MTOR function is differentially regulated in the mouse heart according to the type of stress imposed upon it: with high-intensity treadmill running, MTOR activity became increased, via the activation of its upstream activator Akt, and the heart underwent compensated hypertrophic growth; however, HF-inducing pressure overload inactivated MTOR (4). Moreover, overexpression of active Akt was demonstrated to blunt pressure overload-induced HF development in mice (17), whereas deletion of Akt worsened it (18). Thus, reduced activity of MTOR has an important role in the negative modulation of cardiac function occurring with disease.

To study this role of MTOR, we generated an inducible and cardiac-specific Mtor knockout mouse. In the present report, we demonstrate that these mice develop a fatal, dilated-heart phenotype and that even partial loss of MTOR activity impairs hypertrophic cardiac growth and accelerates HF development. Moreover, there 
A
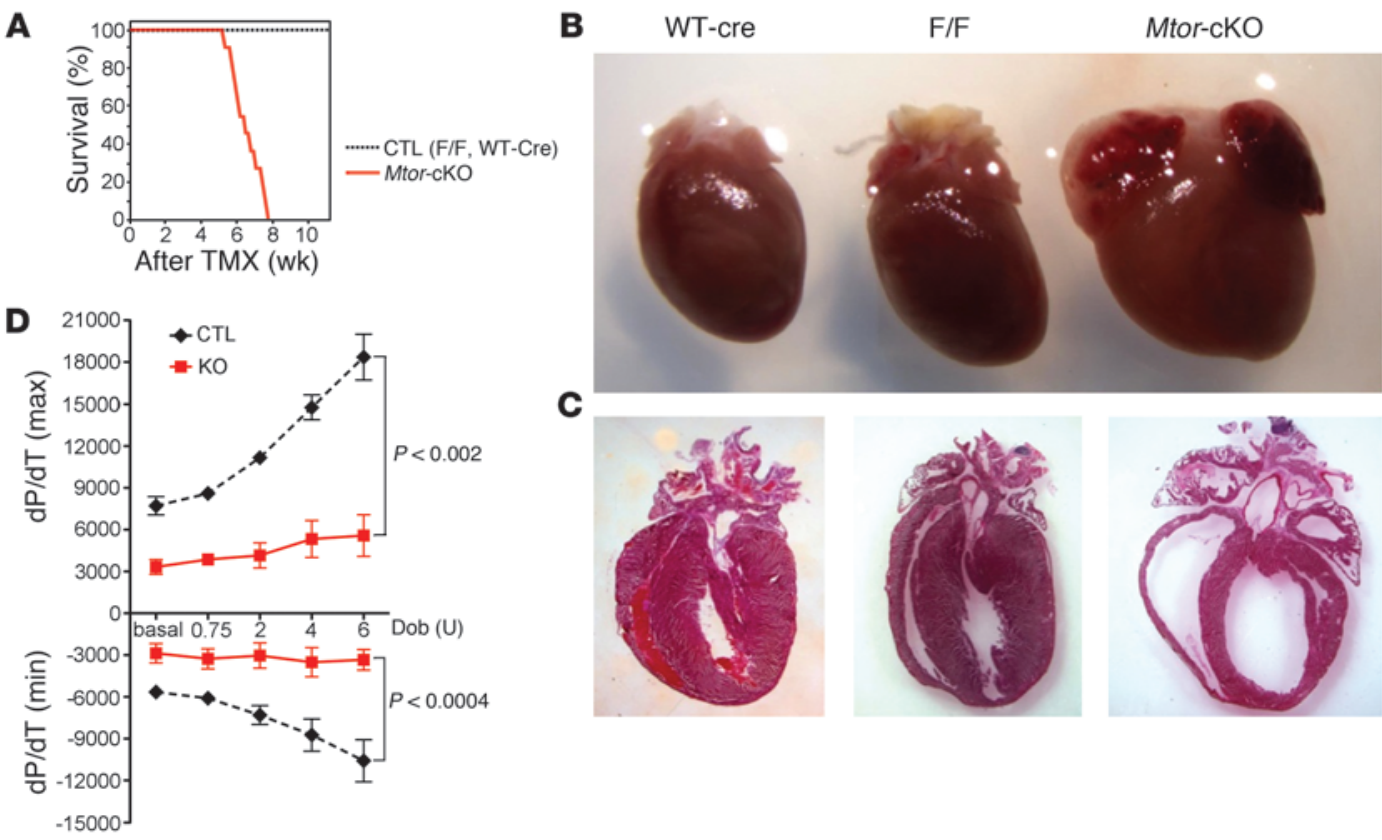

C
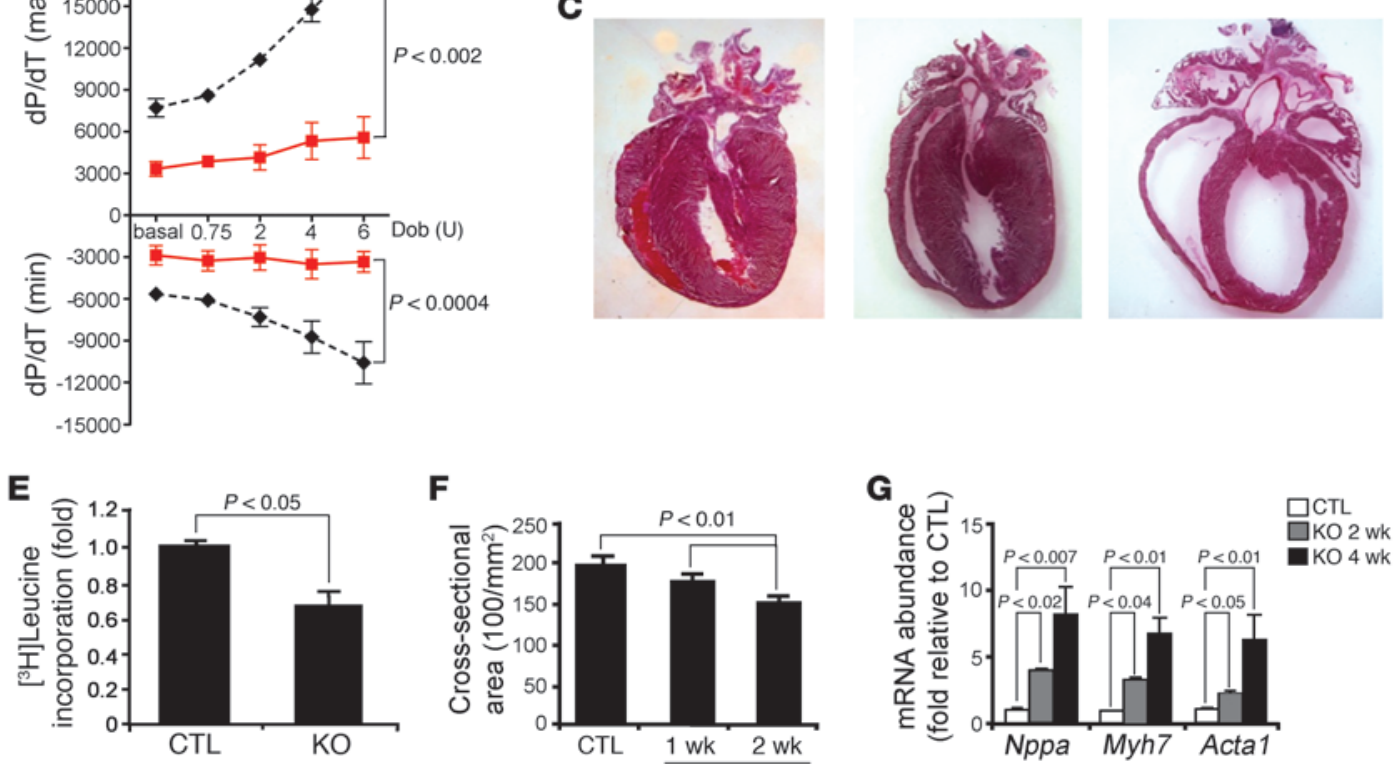

$\mathbf{F}$

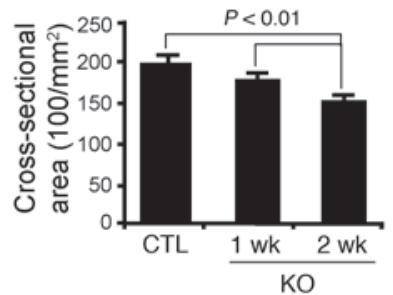

G

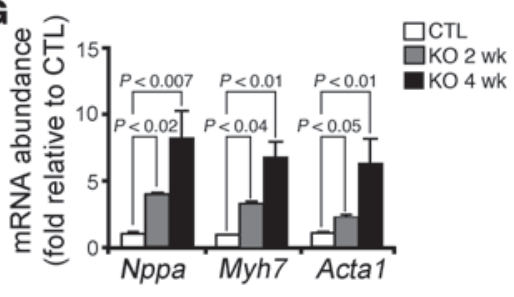

Figure 1

Characterization of Mtor-cKO mice. (A) Kaplan-Meier survival curves of Mtor-cKO and control (CTL) mice. Mortality of Mtor-cKO mice begins 4 weeks after TMX administration and reaches $100 \%$ by 8 weeks. Time is shown as weeks after TMX administration ( $n=11$ for Mtor-cKO mice; $n=7$ for TMX-injected Mtorllfl mice [F/F] control; $n=6$ for WT-Cre control). (B) Representative explanted hearts. The Mtor-cKO heart is larger than that of controls. (C) Representative H\&E-stained heart sections. Mtor-cKO hearts have thinned ventricular walls and enlarged cardiac chambers. (D) Dobutamine stress test, at 4 weeks after TMX administration, of Mtor-cKO (KO) and WT-Cre control hearts. Mtor-cKO hearts have greatly hampered basal inotropy and lusitropy and have only a very weak reactivity to stimulation with increasing units of dobutamine (Dob) (mean $\pm \mathrm{SD} ; n=7$ per group). (E) Incorporation of $\left[{ }^{3} \mathrm{H}\right]$ leucine in cultured adult cardiomyocytes (mean $\pm \mathrm{SD} ; n=3$ ). (F) Fluorescence micrographs of heart tissue were analyzed to obtain cross-sectional cell area (mean $\pm \mathrm{SD}$ ). Cardiomyocytes become significantly and progressively smaller after the administration of TMX. (G) Expression of cardiac fetal genes ( $N p p a$, encoding atrial natriuretic factor; $M y h 7$, encoding $\beta$-myosin heavy chain; Acta1, encoding $\alpha 1$-skeletal actin), assayed by qRT-PCR and normalized for Gapdh expression, is given as fold change. Mtor-cKO hearts have a 2- to 8-fold increase in the expression of these genes with respect to controls at 2 weeks and 4 weeks after TMX administration, respectively (mean $\pm \mathrm{SD}, n=3-4$ per group).

is a remarkable accumulation of dephosphorylated 4E-BP1 in the hearts of Mtor knockout mice, and deletion of the gene encoding 4E-BP1 (Eif4ebp1) improves baseline survival of this model of HF.

\section{Results}

Phenotype characterization of Mtor-cKO mice - Mtor-cKO mice develop a lethal, dilated cardiomyopathy. Because conventional knockout of Mtor results in embryonic death (19-21), we generated an inducible, cardiac-specific Mtor knockout mouse to study the in vivo cardiac function of MTOR in the adult animal. To this end, a gene-targeting strategy was used to generate $\alpha$ MHC-MerCerMer/ Mtorflox/flox mice ( $\alpha$ MHC-MCM/Mtor ${ }^{f / f l}$ mice) (Supplemental Figure $1, \mathrm{~A}-\mathrm{C}$; supplemental material available online with this article; doi:10.1172/JCI43008DS1). Eight-week-old $\alpha$ MHC-MCM/Mtor flflf $^{\prime}$ mice were administered tamoxifen (TMX) for 6 days to induce cardiac-specific knockout of Mtor (Mtor-cKO mice) (Supplemental Figure 1D). MTOR protein expression was decreased 2 weeks after TMX administration and became severely reduced by 4 weeks after TMX (Supplemental Figure 1E and see below). Residual MTOR protein seen after 4 weeks after TMX administration was most likely due to its expression in non-targeted cardiac cells, such as fibroblasts and endothelial cells, as well as in cardiomyocytes, in which an Mtor allele was not recombined. As controls, TMX-injected male mice carrying only one copy of the $\alpha$ MHC-MCM transgene (WTCre mice) were used, unless otherwise stated, in which case TMXinjected Mtor ${ }^{f l / f l}$ mice (F/F mice) or $\alpha$ MHC-MCM/Mtor $r^{f l / f l}$ mice not receiving TMX were used, since there were no differences found at a morphological or histological level or with echocardiography. 
Table 1

Echocardiographic measurements of Mtor-cKO and control mice lines

\begin{tabular}{|c|c|c|c|c|}
\hline Parameter & $\begin{array}{c}\text { Wk after TMX } \\
\text { administration }\end{array}$ & WT-Cre & $F / F$ & Mtor-cKO \\
\hline HR (bpm) & 0 & $535 \pm 67$ & $529 \pm 45$ & $569 \pm 63$ \\
\hline HR (bpm) & 1 & $507 \pm 50$ & $529 \pm 66$ & $554 \pm 50$ \\
\hline HR (bpm) & 2 & $512 \pm 47$ & $578 \pm 47$ & $561 \pm 59$ \\
\hline $\mathrm{HR}$ (bpm) & 4 & $545 \pm 21$ & $572 \pm 70$ & $566 \pm 52$ \\
\hline IVSd (mm) & 0 & $0.59 \pm 0.02$ & $0.62 \pm 0.03$ & $0.61 \pm 0.04$ \\
\hline IVSd (mm) & 1 & $0.60 \pm 0.02$ & $0.61 \pm 0.03$ & $0.61 \pm 0.03$ \\
\hline IVSd (mm) & 2 & $0.61 \pm 0.05$ & $0.61 \pm 0.03$ & $0.6 \pm 0.03$ \\
\hline IVSd (mm) & 4 & $0.57 \pm 0.21$ & $0.61 \pm 0.04$ & $0.54 \pm 0.03^{A}$ \\
\hline IVSs (mm) & 0 & $0.96 \pm 0.07$ & $1.07 \pm 0.07$ & $1.05 \pm 0.13$ \\
\hline IVSs (mm) & 1 & $0.98 \pm 0.06$ & $1.11 \pm 0.07$ & $1.13 \pm 0.09$ \\
\hline IVSs (mm) & 2 & $1.01 \pm 0.08$ & $1.02 \pm 0.06$ & $1.05 \pm 0.07$ \\
\hline IVSs (mm) & 4 & $1.00 \pm 0.05$ & $1.03 \pm 0.07$ & $0.84 \pm 0.10^{\mathrm{A}}$ \\
\hline LVPWd (mm) & 0 & $0.61 \pm 0.03$ & $0.62 \pm 0.04$ & $0.62 \pm 0.04$ \\
\hline LVPWd (mm) & 1 & $0.60 \pm 0.01$ & $0.61 \pm 0.03$ & $0.62 \pm 0.03$ \\
\hline LVPWd (mm) & 2 & $0.59 \pm 0.04$ & $0.60 \pm 0.04$ & $0.59 \pm 0.03$ \\
\hline LVPWd (mm) & 4 & $0.60 \pm 0.02$ & $0.61 \pm 0.03$ & $0.55 \pm 0.03^{A}$ \\
\hline LVPWs (mm) & 0 & $1.05 \pm 0.09$ & $1.20 \pm 0.09$ & $1.17 \pm 0.11$ \\
\hline LVPWs (mm) & 1 & $1.14 \pm 0.07$ & $1.21 \pm 0.07$ & $1.18 \pm 0.11$ \\
\hline LVPWs (mm) & 2 & $1.13 \pm 0.09$ & $1.14 \pm 0.08$ & $1.18 \pm 0.13$ \\
\hline LVPWs (mm) & 4 & $1.09 \pm 0.09$ & $1.15 \pm 0.07$ & $0.89 \pm 0.13^{\mathrm{A}}$ \\
\hline LVIDd (mm) & 0 & $3.31 \pm 0.12$ & $3.59 \pm 0.24$ & $3.36 \pm 0.44$ \\
\hline LVIDd (mm) & 1 & $3.42 \pm 0.37$ & $3.75 \pm 0.41$ & $3.61 \pm 0.29$ \\
\hline LVIDd (mm) & 2 & $3.21 \pm 0.27$ & $3.41 \pm 0.25$ & $3.24 \pm 0.28$ \\
\hline LVIDd (mm) & 4 & $2.86 \pm 0.28$ & $3.51 \pm 0.46$ & $4.06 \pm 0.37^{A}$ \\
\hline LVIDs (mm) & 0 & $1.65 \pm 0.21$ & $1.9 \pm 0.36$ & $1.73 \pm 0.37$ \\
\hline LVIDs (mm) & 1 & $1.76 \pm 0.35$ & $1.92 \pm 0.48$ & $2.00 \pm 0.22$ \\
\hline LVIDs (mm) & 2 & $1.81 \pm 0.31$ & $1.73 \pm 0.24$ & $1.71 \pm 0.25$ \\
\hline LVIDs (mm) & 4 & $1.47 \pm 0.26$ & $1.83 \pm 0.36$ & $3.09 \pm 0.51^{A}$ \\
\hline LVFS (\%) & 0 & $47.3 \pm 5.6$ & $47.3 \pm 7.4$ & $46.1 \pm 7.7$ \\
\hline LVFS (\%) & 1 & $45.5 \pm 5.8$ & $49.3 \pm 8.4$ & $44.6 \pm 3.8$ \\
\hline LVFS (\%) & 2 & $47.2 \pm 6.4$ & $49.4 \pm 5.5$ & $46.7 \pm 8.8$ \\
\hline LVFS (\%) & 4 & $49.2 \pm 5.2$ & $46.8 \pm 5.5$ & $26.4 \pm 6.7^{A}$ \\
\hline
\end{tabular}

HR, heart rate; IVSd, intraventricular septal thickness in diastole; IVSs, intraventricular septal thickness in systole; LVPWd, LV posterior wall thickness in diastole; LVPWs, LV posterior wall thickness in systole; LVIDd, LV internal diameter in diastole; LVIDs, LV internal diameter in systole; LVFS, LV fractional shortening. $n=6$ mice assessed in the WT-Cre control group, $n=9$ mice assessed in the F/F control group, and $n=9$ mice assessed in the Mtor-cKO group. Measurements are given as mean \pm SD. ${ }^{A} P<0.05$ versus control groups.

Mtor-cKO mice started dying at 4 weeks after TMX administration: the median survival age was 6 weeks after TMX administration, and none of the mice survived for longer than 8 weeks (Figure 1A). Mtor-cKO mice presented with pleural effusions and ascites but had no significant abnormalities in liver, kidney, or skeletal muscle (data not shown). However, at 4 weeks after TMX administration, the Mtor-cKO heart was significantly enlarged compared with that from controls (Figure 1B and Supplemental Figure 1F). Atrial and ventricular chambers were enlarged, and the LV wall and interventricular septum were thinner than normal (Table 1, Figure 1C, and Supplemental Figure 1G). Cardiac function assessed using echocardiography (Table 1) and the dobutamine stress test (Figure 1D) evidenced frank HF at 4 weeks after TMX administration.

Incorporation of $\left[{ }^{3} \mathrm{H}\right]$ leucine, a qualitative assay for protein synthesis, was significantly reduced in cultured adult Mtor-cKO cardiomyocytes compared with those of controls (Figure 1E). The cross-sectional area of Mtor-cKO cardiomyocytes was progressively reduced (Figure 1F and Supplemental Figure 2A), and isolated adult cardiomyocytes were significantly thinner and shorter than those of controls (Supplemental Figure 2, B and C). We did not observe any increase of proliferation in Mtor-cKO heart (Supplemental Figure 2D). The decrease in the rate of protein synthesis, which is the most energy-demanding cellular process, suggests that in this model energy supply is not a key element in the determination of HF; however, changes in the metabolism of energyproviding substrates were not addressed here.

In addition, Mtor-cKO myocardium expressed progressively higher levels of mRNA for atrial natriuretic peptide, $\beta$-myosin heavy chain, and $\alpha 1$-skeletal actin, 3 fetal genes that are characteristically reexpressed when the heart is subjected to pathological stress (22) (Figure 1G).

Cell death and fibrosis are enhanced, and mitochondria are compromised, in Mtor-cKO mice. TUNEL staining revealed that the number of apoptotic cardiomyocytes increased progressively and dramatically after TMX administration (Figure 2A and Supplemental Figure $3 \mathrm{~A})$. Cleaved poly(ADP-ribose) polymerase (Parp), another apoptotic marker, was significantly increased at 4 weeks after TMX administration (Figure 2B). Moreover, cytochrome $c$ oxidase subunit IV (CoxIV) expression became progressively reduced (Figure 2C), indicating a loss in the oxidative capacity of mitochondria in Mtor-cKO myocardium (23). Mitochondrial structure was affected early on, by 2 weeks after TMX administration (Figure 2D). Therefore, mitochondrial function is rapidly compromised by the absence of MTOR, inducing apoptosis of cardiomyocytes.

Longitudinal heart sections stained with Malloy's trichrome revealed the presence of large spaces between cardiomyocytes - indicative of tissue edema - and interstitial fibrosis in the LV and interventricular septum of Mtor-cKO mice (Supplemental Figure 3B). Fibrosis increased progressively after TMX administration (Figure 2E).

Because MTOR inhibits autophagy, we examined whether gene products of the autophagic pathway changed in this model. We found an increase of Atg6/beclin-1, Atg7, Atg8/LC3B (LC3BI-II), and Atg12 (Figure 2F), key components of the lysosomal machinery. Foxo1 and Foxo3a, transcription factors involved in regulation of autophagy, became increasingly expressed in the myocardium of Mtor-cKO mice (Figure $3 \mathrm{~B}$ ). In addition, electron microscopy revealed the presence of autophagic bodies (Figure 2G). These results suggest that autophagy is increased in Mtor-cKO myocardium.

Knockout of MTOR is accompanied by upregulation of 4E-BP1. To delineate the molecular mechanisms involved in producing the phenotypic characteristics of Mtor-cKO mice, we determined the activity of MTORC1 and MTORC2, the 2 multiprotein complexes containing MTOR. We first focused on 2 target proteins, 4E-BP1 and p70S6 kinase 1 and its substrate S6 (S6K1/S6), main downstream effectors of MTORC1 (Figure 3A). 4E-BP1 became progressively more expressed up to 4 weeks after TMX administration and remained elevated thereafter; moreover, it became increasingly dephosphory- 
A

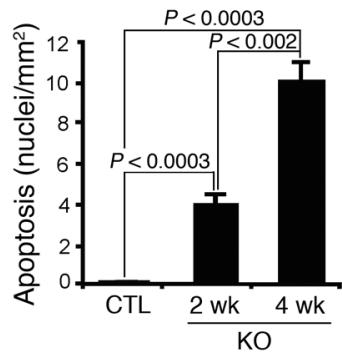

D
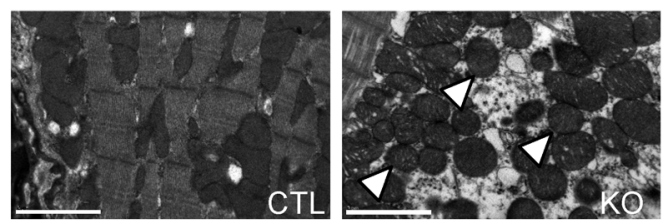

B
F

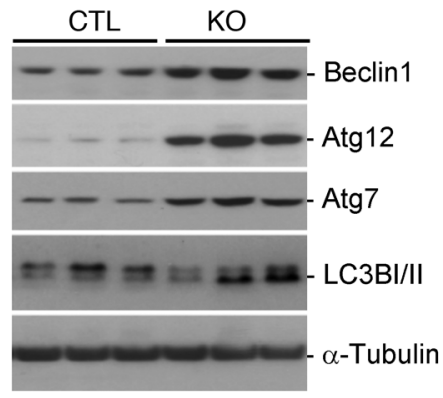

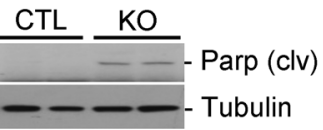

C
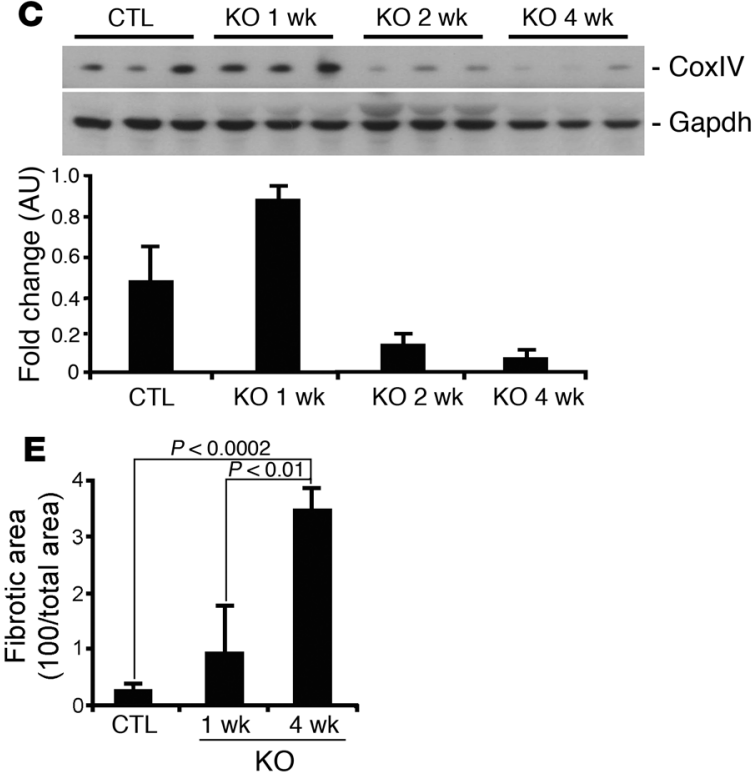

G

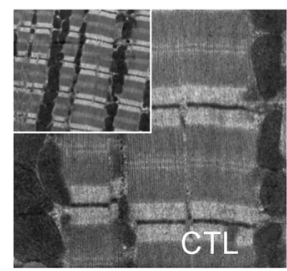

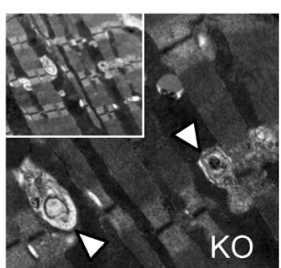

Figure 2

Analysis of cell death. (A) Image analysis of TUNEL-stained sections revealed that the Mtor-cKO heart is subjected to a progressive loss of cardiomyocytes because of the induction of apoptosis (mean \pm SD; $n=3$ per group). (B) Representative Western blot for cleaved (clv) Parp. Cleaved Parp expression is increased in the heart of Mtor-cKO mice with respect to WT-Cre control littermates at 4 weeks after TMX administration. $\alpha$-Tubulin was used as an internal loading control. (C) Western blot for CoxIV expression (top). Gapdh was used as an internal loading control. Densitometric analysis of CoxIV expression (mean \pm SD) in extracts from WT-Cre controls at 4 weeks after TMX administration and from Mtor-cKO mice at 1, 2, and 4 weeks after TMX administration (bottom). (D) Representative transmission electron micrographs of Mtor-cKO and WT-Cre control hearts, both at 2 weeks after TMX administration. Mtor-cKO LV myocardium presents with sarcomeric disarray and swollen mitochondria (arrowheads), whereas that of control has a normal ultrastructure. Original magnification: $\times 2,000$. (E) Image analysis reveals that the fibrotic index of the Mtor-cKO heart is increased already at 1 week after TMX administration and becomes significantly increased thereafter (mean $\pm \mathrm{SD}, n=3$ per group). (F) Western blot of some autophagy-related proteins. Expression of the 4 proteins analyzed is increased in the Mtor-cKO heart with respect to that of the WT-Cre control at 1 week after TMX administration. (G) Representative transmission electron micrographs of WT-Cre control and Mtor-cKO heart, both at 1 week after TMX administration. Autophagic bodies (arrowheads) were observed in Mtor-cKO myocardium. Original magnification: $\times 4,000$; inset, $\times 2,000$.

lated. The increased expression of 4E-BP1 protein was accompanied by an increased level of its mRNA (Supplemental Figure 3C). Phosphorylation levels of ribosomal protein S6K1 and of its substrate S6 decreased below control levels by week 2 after TMX administration. Phosphorylation levels of insulin receptor substrate 1 (Irs1) and total Irs1 protein content increased with the decreased phosphorylation of the Irs1 inhibitor, S6K (24) (Figure 3A), similarly to results described for skeletal muscle $(23,25)$. In addition, expression of PRAS40, a negative regulator of MTORC1, was progressively increased in Mtor-cKO myocardium.

We then focused on Akt and PKC- $\alpha, 2$ MTORC2 substrates. Phosphorylation of Akt at Ser473 was increased from week 1 through to week 4 after TMX administration (Figure 3B). This is surprising, considering that MTORC2 is a major Ser473 kinase (15) and that MTOR becomes progressively downregulated, and may indicate the presence of another kinase with specificity for this substrate besides mTORC2, as also indicated by others $(23,25)$. In addition,
Akt was increasingly phosphorylated at residue Thr308; this may be ascribed to the increase in Irs1 activity following the inhibition of S6K and a consequential increase in Pdk1 activity. On the other hand, phosphorylation at the Thr450 site of Akt remained at baseline levels, decreasing only at 4 weeks after TMX administration.

$\mathrm{PKC}-\alpha$ protein expression, which reflects its phosphorylation state and stabilization by MTORC2 (12), remained constant up to 2 weeks after TMX administration but decreased from then on (Figure 3B), following the decrease of Rictor and MTOR protein levels. Moreover, downregulation of Rictor had a different time course than that of Raptor, with Raptor becoming more rapidly degraded compared with Rictor (Figure 3B).

To determine whether decreased MTOR activity is associated with HF, we studied the transverse aortic constriction (TAC) model of pressure overload. We found that after 8 weeks of TAC - when mice are in frank HF (17) - the phosphorylation of downstream targets of MTORC1 was reduced (Figure 3C). Moreover, there was 
A
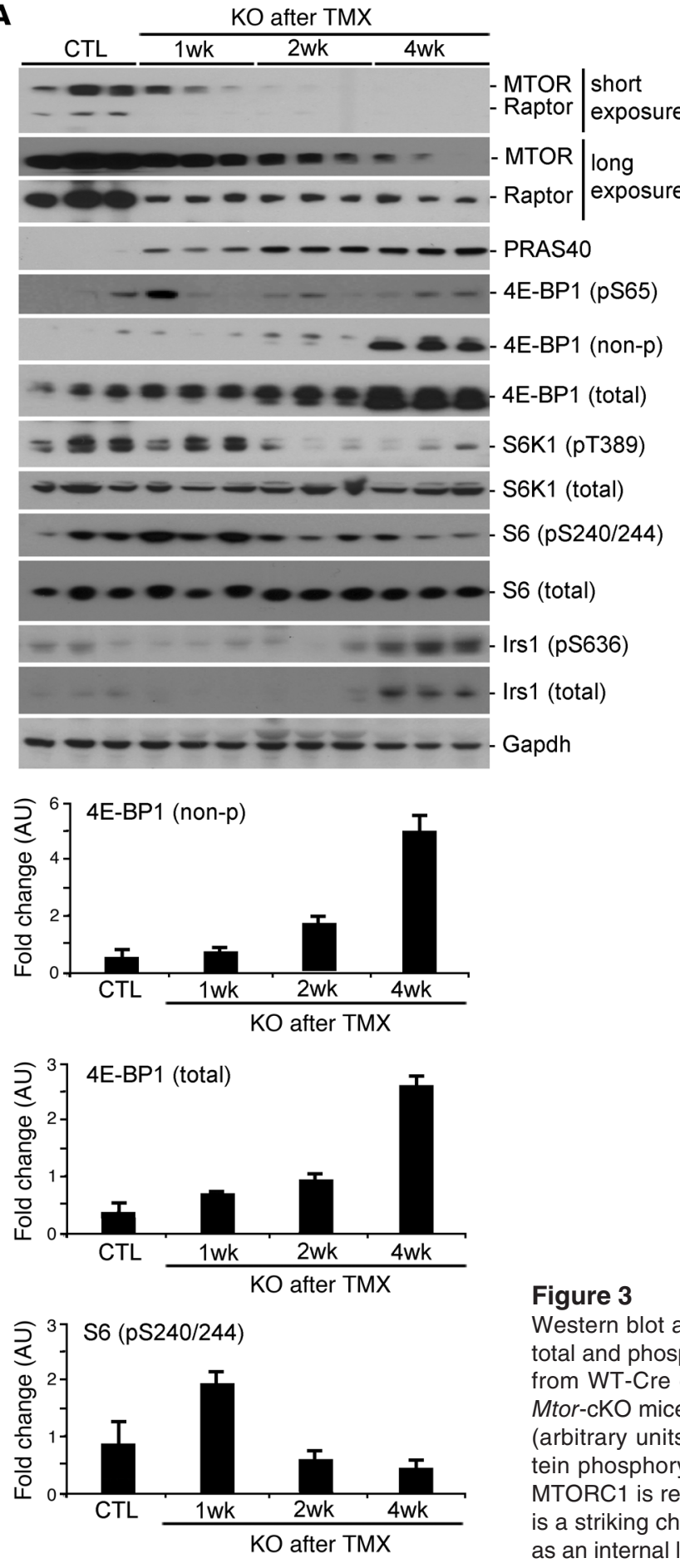

\section{Figure 3}

B
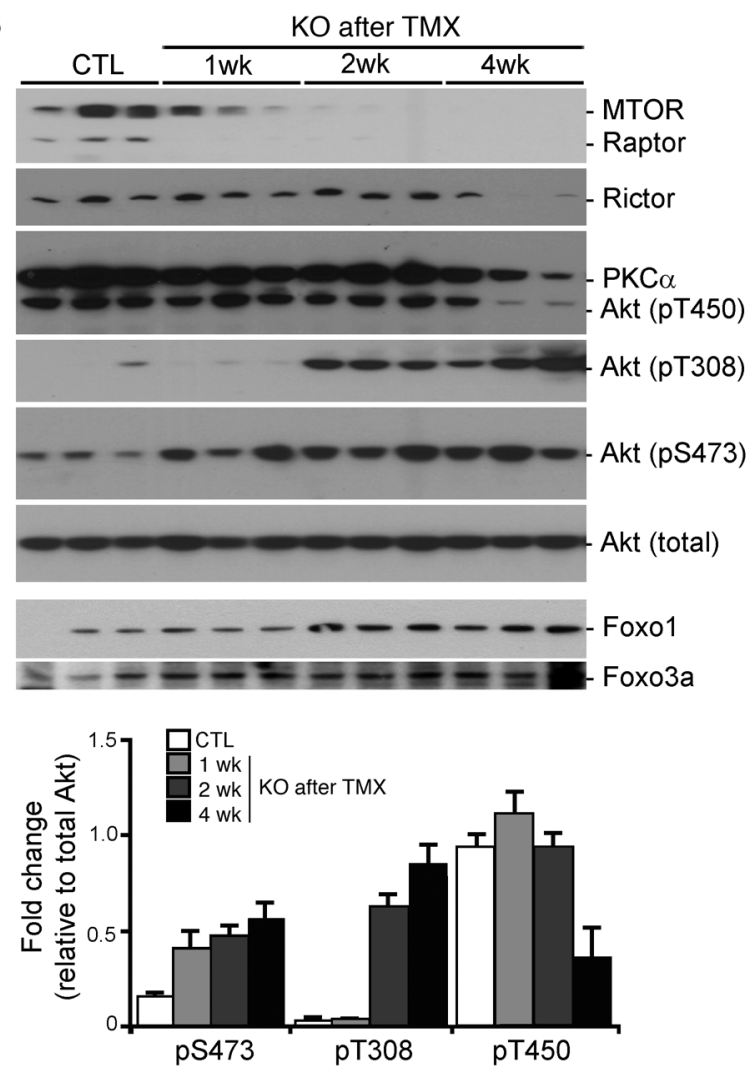

C

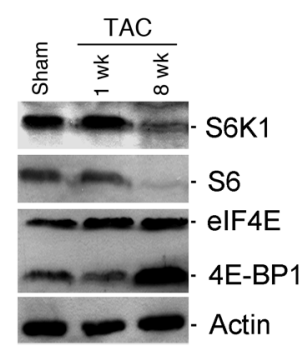

D

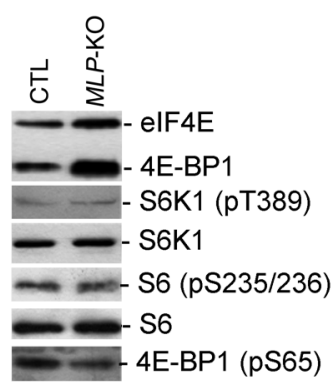

Western blot analysis of MTORC1- and MTORC2-associated proteins. (A) Blots of total and phosphorylated (p) MTORC1-associated proteins from myocardial extracts from WT-Cre controls at 4 weeks after TMX administration (post-TMX) and from Mtor-cKO mice at 1, 2, and 4 weeks after TMX administration (above). Densitometry (arbitrary units, mean \pm SD) of nonphosphorylated and total 4E-BP1 and S6 protein phosphorylated at Ser240 (below). Phosphorylation of downstream targets of MTORC 1 is reduced by 2 weeks after TMX administration. Accumulation of 4E-BP1 is a striking characteristic of myocardium lacking Mtor expression. Gapdh was used as an internal loading control. (B) Blots of total and phosphorylated MTORC2-associated proteins and of Foxo proteins in myocardial extracts from WT-Cre controls at 4 weeks after TMX administration and from Mtor-cKO mice at 1, 2, and 4 weeks after TMX administration (top). Phosphorylation of Akt at sites independent of MTORC2 (Thr308 and Thr450) was also analyzed. Densitometry (arbitrary units, mean \pm SD) of phosphorylation levels of Akt (bottom). (C) Representative blot of myocardial extracts from sham- and TAC-operated wild-type mice. The MTORC1 downstream proteins S6 kinase and $\mathrm{S} 6$ become less expressed when hearts are failing ( 8 weeks after TAC). Note the accumulation of 4E-BP1 bound to elF4E at 8 weeks in TAC-operated mice. (D) Representative blot of myocardial extracts from wild-type littermates and $M L P-K O$ mice, a genetic model of dilated cardiomyopathy. Similarly to Mtor-cKO (A) and failing pressure-overloaded wild-type myocardium (C), 4E-BP1 becomes dephosphorylated at Ser65 and more bound to elF4E also in this model. 
Table 2

Echocardiographic measurements of Mtor-cKO and control mice before TAC and 2 weeks after TAC

\begin{tabular}{|c|c|c|c|c|c|c|c|c|}
\hline \multirow[t]{3}{*}{ Parameter } & \multicolumn{4}{|c|}{ No TAC } & \multicolumn{4}{|c|}{ TMX (3 weeks) } \\
\hline & \multicolumn{2}{|c|}{ - } & \multicolumn{2}{|c|}{ TMX (1 week) } & \multicolumn{2}{|c|}{ Sham } & \multicolumn{2}{|c|}{ TAC } \\
\hline & WT-Cre & Mtor-cKO & WT-Cre & Mtor-cKO & WT-Cre & Mtor-cKO & WT-Cre & Mtor-cKO \\
\hline HR (bpm) & $530 \pm 44$ & $540 \pm 51$ & $574 \pm 66$ & $554 \pm 52$ & $578 \pm 47$ & $561 \pm 56$ & $600 \pm 27$ & $571 \pm 44$ \\
\hline IVSd (mm) & $0.62 \pm 0.02$ & $0.62 \pm 0.04$ & $0.63 \pm 0.03$ & $0.61 \pm 0.02$ & $0.62 \pm 0.04$ & $0.60 \pm 0.03$ & $0.89 \pm 0.10^{\mathrm{A}}$ & $0.70 \pm 0.11$ \\
\hline IVSs (mm) & $1.09 \pm 0.07$ & $1.05 \pm 0.03$ & $1.14 \pm 0.09$ & $1.10 \pm 0.07$ & $1.02 \pm 0.08$ & $1.03 \pm 0.03$ & $1.37 \pm 0.09^{A}$ & $1.15 \pm 0.20$ \\
\hline LVPWd (mm) & $0.61 \pm 0.03$ & $0.64 \pm 0.04$ & $0.61 \pm 0.01$ & $0.62 \pm 0.02$ & $0.60 \pm 0.04$ & $0.59 \pm 0.02$ & $0.93 \pm 0.10^{\mathrm{A}}$ & $0.72 \pm 0.12^{\mathrm{B}}$ \\
\hline LVPWs (mm) & $1.23 \pm 0.07$ & $1.22 \pm 0.13$ & $1.19 \pm 0.04$ & $1.16 \pm 0.13$ & $1.13 \pm 0.07$ & $1.20 \pm 0.08$ & $1.36 \pm 0.15$ & $1.24 \pm 0.23$ \\
\hline LVIDd (mm) & $3.75 \pm 0.17$ & $3.38 \pm 0.17$ & $3.39 \pm 0.24$ & $3.61 \pm 0.33$ & $3.41 \pm 0.31$ & $3.24 \pm 0.14$ & $2.96 \pm 0.42$ & $3.78 \pm 0.50^{\mathrm{B}}$ \\
\hline LVIDs (mm) & $2.02 \pm 0.4$ & $1.66 \pm 0.24$ & $1.61 \pm 0.2$ & $1.96 \pm 0.26$ & $1.74 \pm 0.28$ & $1.70 \pm 0.31$ & $1.44 \pm 0.27$ & $2.48 \pm 0.73^{B}$ \\
\hline LVFS $(\%)$ & $47.3 \pm 7.3$ & $48.1 \pm 6.0$ & $49.4 \pm 8.9$ & $47.0 \pm 4.0$ & $49.5 \pm 7.0$ & $46.7 \pm 9.2$ & $51.0 \pm 8.6$ & $35.5 \pm 10.3^{B, C}$ \\
\hline
\end{tabular}

The "-" symbol indicates mice not administered TMX. $n=5$ WT-Cre and Mtor-cKO mice not subjected to TAC/sham, $n=12$ WT-Cre and Mtor-cKO mice subjected to TAC/sham. Measurements are given as mean $\pm \mathrm{SD}$. ${ }^{\mathrm{A}} P<0.05$ versus WT-Cre sham mice; ${ }^{\mathrm{B}} P<0.05$ versus $\mathrm{WT}-\mathrm{C}$ re $\mathrm{TAC}$ mice; $\mathrm{C} P<0.05$ versus Mtor-cKO sham mice.

an increase in the amount of 4E-BP1 bound to eIF4E, as determined by $\mathrm{m}^{7} \mathrm{GTP}$-sepharose chromatography. We also found increased binding of 4E-BP1 to eIF4E in the heart of the muscle LIM protein knockout mouse (MLP-KO mouse), a genetic model of dilated cardiomyopathy (26) (Figure 3D). Therefore, reduced MTOR activity and a consequent increased inhibitory activity of 4E-BP1 seem broadly associated with the development of overt HF.

MTOR-dependent heart bypertrophy is required to cope with pressure overload stress. The notion that hypertrophy is beneficial for cardiac function under pathologic conditions has been challenged in recent years (27). Because up to $2 / 3$ weeks after TMX administration the MTOR protein level in Mtor-cKO myocardium is decreased but not totally absent and cardiac function is maintained, we reasoned that this model could be used to determine (a) whether a decreased level of MTOR protein affects the hypertrophic response to pressure overload-induced hypertrophy, and (b) if this response was found to be hampered, whether the blunting of hypertrophy is in fact beneficial for heart function in a pathological setting. We therefore subjected Mtor-cKO mice to TAC at 1 week after TMX administration and assessed cardiac function for the following 2 weeks (Supplemental Figure 4A).

We found that 2 weeks after the TAC procedure (i.e., 3 weeks after TMX administration) Mtor-cKO mice had a significantly reduced increase in LV wall thickness and a more dilated LV chamber compared with control TAC mice (Table 2). The ratios of LV weightand heart weight-to-tibial length were significantly reduced in Mtor-cKO-TAC mice (Supplemental Figure 4B). The significant worsening of contractile function observed in Mtor-cKO mice was accelerated (Table 2), and there was a diminished induction of fetal genes (Figure 4A). Moreover, survival of Mtor-cKO mice was further significantly reduced (Supplemental Figure 4C). Thus, even a partial reduction in MTOR expression markedly affects the development of pressure overload-induced hypertrophy and the ability of the heart to maintain a compensatory function, accelerating HF progression and death.

At the biochemical level, total 4E-BP1 was dramatically increased in Mtor-cKO mice subjected to TAC with respect to that of control TAC mice. Furthermore, phosphorylation of 4E-BP1 and of S6
$\mathbf{A}$

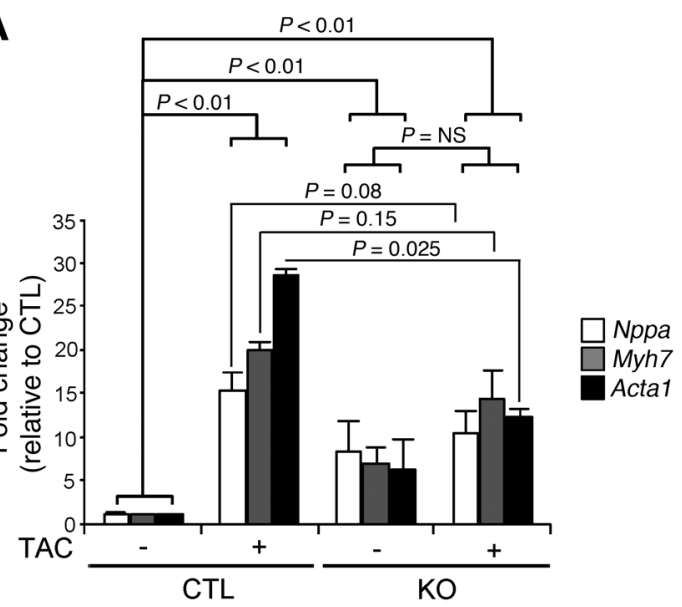

B

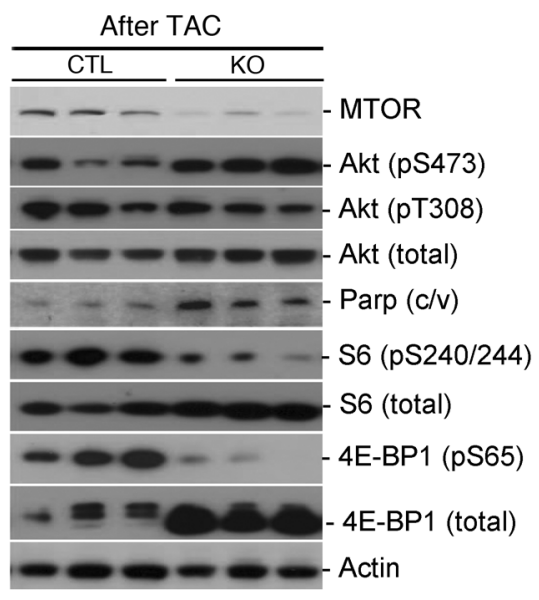

Figure 4

Pressure overload stress in Mtor-cKO mice. (A) qRT-PCR analysis for cardiac fetal gene expression, normalized for Gapdh, involved in the response to TAC in WT-Cre control and Mtor-cKO hearts. After 3 weeks of TAC (4 weeks after TMX administration for both), there is only a moderate increase in the expression of these genes in Mtor-cKO hearts with respect to the similarly treated control group (mean \pm SD, $n=3-4$ per group). (B) Representative Western blots of TAC-operated WT-Cre and Mtor-cKO hearts, 3 weeks after the surgery. 
A

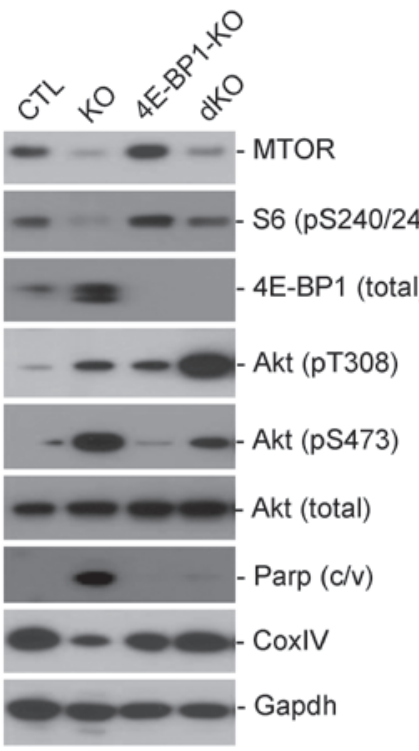

B

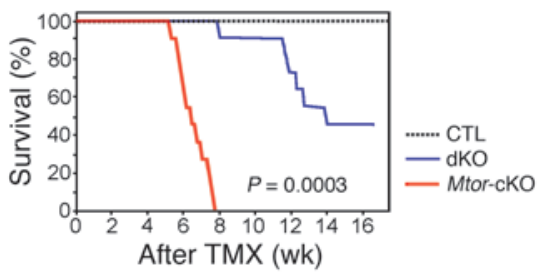

\section{C}

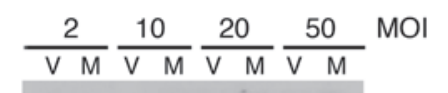

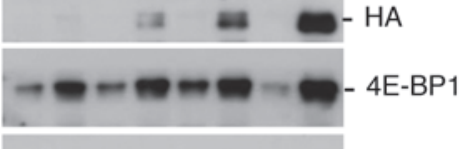
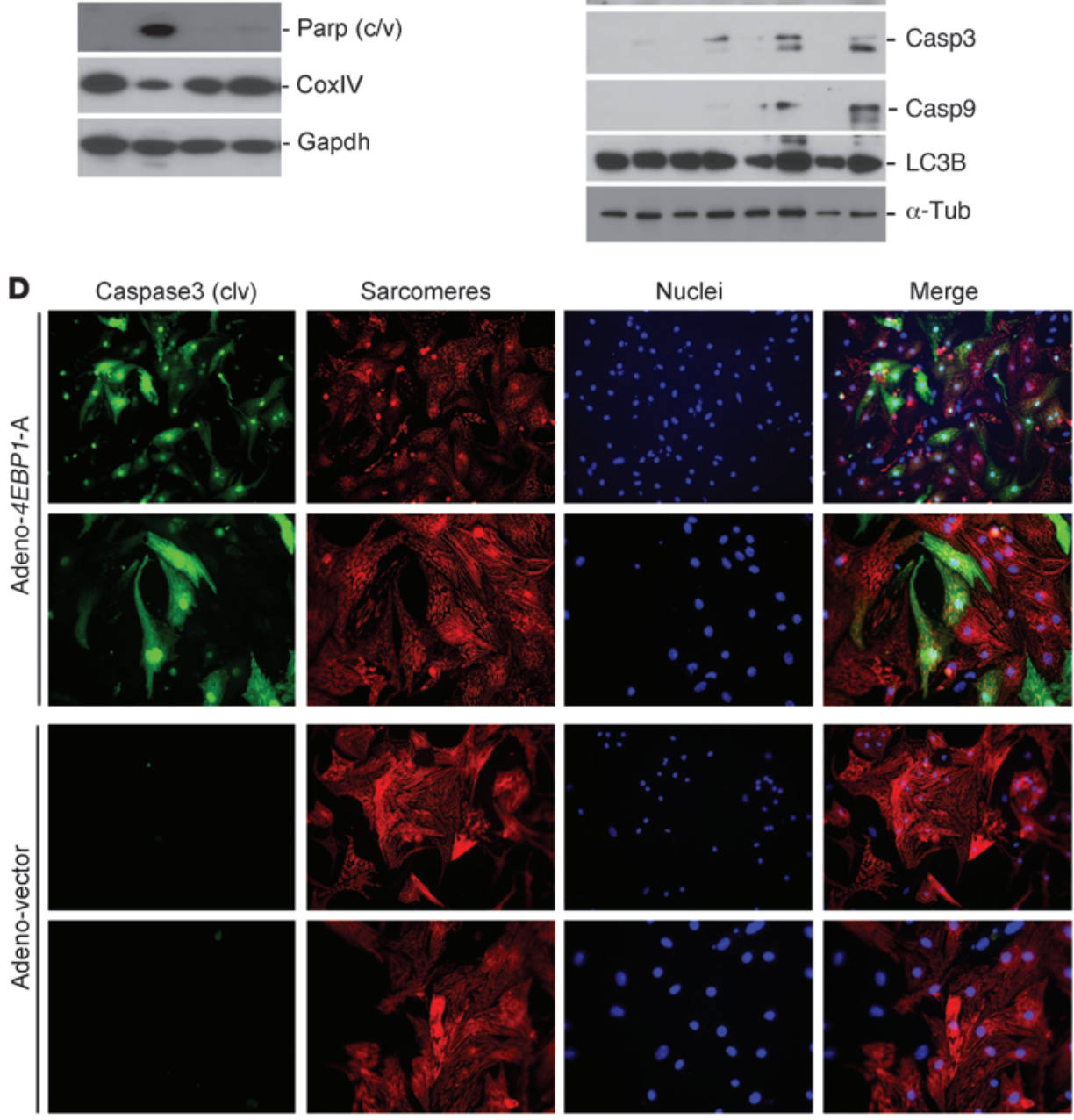

\section{Figure 5}

Survival of Mtor-cKO mice is improved by deletion of Eif4ebp1, and hypophosphorylated 4E-BP1 promotes apoptosis in cardiomyocytes. (A) Representative Western blots, at 4 weeks after TMX administration, of WT-Cre, Mtor-cKO, 4E-BP1-KO, and Mtor/4E-BP1 dKO mouse heart lysates. (B) Kaplan-Meier survival curves of Mtor/4E-BP1 dKO $(n=13)$, Mtor-cKO $(n=10)$, and WTCre mice $(n=9)$. Survival of Mtor/4EBP1 dKO mice is significantly better than that of Mtor-cKO mice. (C) Representative Western blots demonstrating progressive increase of cleaved caspase 3 (Casp3) and caspase 9 in lysates obtained from cultured neonatal cardiomyocytes after 24-hour infection with adenovirus carrying a mutated (hypophosphorylated) form of 4E-BP1 (4E-BP1-5A). V, empty vector; $M$, transgene-carrying vector; $H A$, hemagglutinin tag; Tub, $\alpha$-tubulin. (D) Immunofluorescence of infected cardiomyocytes. Green indicates cleaved caspase 3; red indicates $\alpha$-actinin; blue indicates Hoechst staining of nuclei. Original magnification: $\times 20$ (rows 1 and 3); ×40 (rows 2 and 4). was reduced, indicating a significant decrease of MTORC1 activity. Phosphorylation of Akt at Ser473 remained elevated, whereas it became deceased at Thr308. Moreover, cleaved Parp was significantly increased in Mtor-cKO-TAC myocardium, suggestive of increased apoptosis (Figure 4B).

As a whole, these data suggest that MTOR, and in particular MTORC1, plays an essential role in the response of the heart to stress by preserving cardiac function through the induction of hypertrophy. Ablation of Eif4ebp1 significantly improves HF in Mtor-cKO mice. Previous work demonstrated that the absence of S6K1 and S6K2 was not associated with any effect on the ability of the heart to undergo hypertrophy after pressure overload (28). In contrast, the role of 4E-BP1 in modulating cardiac function has not been assessed yet in mammals. To determine whether accumulation of dephosphorylated 4E-BP1 is linked to HF onset, we crossed Mtor-cKO mice with Eif4ebp1 knockout mice (4E-BP1-KO mice) (29) to generate a double-KO ( $\mathrm{dKO})$ model. 4E-BP1 is the most expressed 4E-BP in striated and cardiac muscle (29) (Supplemental Figure 3D); its deletion caused an increase in $\left[{ }^{3} \mathrm{H}\right]$ leucine uptake in the heart (Supplemental Figure 5A) and a significant 
Table 3

Echocardiographic measurements of the various mouse lines used in the study

\begin{tabular}{|c|c|c|c|c|c|}
\hline Parameter & $\begin{array}{l}\text { Wk after TMX } \\
\text { administration }\end{array}$ & WT-Cre & Mtor-cKO & 4ЕВP1-KO & dKO \\
\hline HR (bpm) & 0 & $540 \pm 68$ & $569 \pm 59$ & $563 \pm 54$ & $529 \pm 73$ \\
\hline HR (bpm) & 1 & $576 \pm 90$ & $554 \pm 56$ & $567 \pm 38$ & $551 \pm 53$ \\
\hline $\mathrm{HR}$ (bpm) & 2 & $600 \pm 62$ & $561 \pm 56$ & $598 \pm 53$ & $613 \pm 35$ \\
\hline $\mathrm{HR}$ (bpm) & 4 & $606 \pm 47$ & $566 \pm 52$ & $622 \pm 29$ & $609 \pm 66$ \\
\hline IVSd (mm) & 0 & $0.61 \pm 0.03$ & $0.63 \pm 0.04$ & $0.58 \pm 0.03$ & $0.58 \pm 0.03$ \\
\hline IVSd (mm) & 1 & $0.61 \pm 0.03$ & $0.62 \pm 0.02$ & $0.55 \pm 0.02$ & $0.58 \pm 0.04$ \\
\hline IVSd (mm) & 2 & $0.62 \pm 0.03$ & $0.60 \pm 0.02$ & $0.61 \pm 0.02$ & $0.6 \pm 0.02$ \\
\hline IVSd (mm) & 4 & $0.61 \pm 0.03$ & $0.55 \pm 0.03^{A}$ & $0.61 \pm 0.02$ & $0.62 \pm 0.03^{B}$ \\
\hline IVSs (mm) & 0 & $1.05 \pm 0.06$ & $1.04 \pm 0.11$ & $0.98 \pm 0.05$ & $0.99 \pm 0.09$ \\
\hline IVSs (mm) & 1 & $1.07 \pm 0.09$ & $1.12 \pm 0.09$ & $0.96 \pm 0.06$ & $1.05 \pm 0.06$ \\
\hline IVSs (mm) & 2 & $1.04 \pm 0.06$ & $1.04 \pm 0.08$ & $1.04 \pm 0.04$ & $1.01 \pm 0.06$ \\
\hline IVSs (mm) & 4 & $1.04 \pm 0.07$ & $0.88 \pm 0.12^{\mathrm{A}}$ & $1.04 \pm 0.05$ & $1.09 \pm 0.05$ \\
\hline LVPWd (mm) & 0 & $0.62 \pm 0.03$ & $0.62 \pm 0.04$ & $0.58 \pm 0.02$ & $0.56 \pm 0.03$ \\
\hline LVPWd (mm) & 1 & $0.60 \pm 0.04$ & $0.62 \pm 0.03$ & $0.54 \pm 0.02$ & $0.59 \pm 0.02$ \\
\hline LVPWd (mm) & 2 & $0.61 \pm 0.04$ & $0.59 \pm 0.03$ & $0.60 \pm 0.03$ & $0.60 \pm 0.01$ \\
\hline LVPWd (mm) & 4 & $0.60 \pm 0.03$ & $0.55 \pm 0.03^{A}$ & $0.61 \pm 0.02$ & $0.62 \pm 0.03^{B}$ \\
\hline LVPWs (mm) & 0 & $1.18 \pm 0.10$ & $1.17 \pm 0.10$ & $1.10 \pm 0.11$ & $1.07 \pm 0.08$ \\
\hline LVPWs (mm) & 1 & $1.18 \pm 0.07$ & $1.18 \pm 0.10$ & $1.07 \pm 0.09$ & $1.18 \pm 0.09$ \\
\hline LVPWs (mm) & 2 & $1.14 \pm 0.07$ & $1.16 \pm 0.14$ & $1.18 \pm 0.11$ & $1.13 \pm 0.10$ \\
\hline LVPWs (mm) & 4 & $1.16 \pm 0.08$ & $0.92 \pm 0.14^{A}$ & $1.19 \pm 0.09$ & $1.27 \pm 0.04^{B}$ \\
\hline LVIDd (mm) & 0 & $3.49 \pm 0.35$ & $3.36 \pm 0.39$ & $2.87 \pm 0.16$ & $2.94 \pm 0.44$ \\
\hline LVIDd (mm) & 1 & $3.58 \pm 0.45$ & $3.61 \pm 0.30$ & $3.14 \pm 0.17$ & $3.08 \pm 0.17$ \\
\hline LVIDd (mm) & 2 & $3.42 \pm 0.24$ & $3.24 \pm 0.28$ & $3.08 \pm 0.42$ & $2.95 \pm 0.44$ \\
\hline LVIDd (mm) & 4 & $3.48 \pm 0.41$ & $4.06 \pm 0.43^{A}$ & $3.25 \pm 0.32$ & $3.38 \pm 0.30^{B}$ \\
\hline LVIDs (mm) & 0 & $1.87 \pm 0.37$ & $1.81 \pm 0.35$ & $1.48 \pm 0.16$ & $1.49 \pm 0.34$ \\
\hline LVIDs (mm) & 1 & $1.83 \pm 0.42$ & $2.00 \pm 0.21$ & $1.69 \pm 0.14$ & $1.69 \pm 0.15$ \\
\hline LVIDs (mm) & 2 & $1.74 \pm 0.24$ & $1.72 \pm 0.24$ & $1.49 \pm 0.33$ & $1.57 \pm 0.32$ \\
\hline LVIDs (mm) & 4 & $1.84 \pm 0.35$ & $3.01 \pm 0.59 \mathrm{~A}$ & $1.71 \pm 0.27$ & $1.81 \pm 0.25^{B}$ \\
\hline LVFS (\%) & 0 & $46.6 \pm 6.7$ & $46.1 \pm 7.2$ & $48.4 \pm 4.7$ & $49.7 \pm 5.5$ \\
\hline LVFS (\%) & 1 & $49.1 \pm 6.9$ & $44.6 \pm 4.7$ & $46.1 \pm 3.6$ & $45.3 \pm 4.4$ \\
\hline LVFS ( $\%)$ & 2 & $49.1 \pm 5.1$ & $46.7 \pm 8.5$ & $51.9 \pm 5.8$ & $47.0 \pm 5.4$ \\
\hline LVFS (\%) & 4 & $47.4 \pm 5.1$ & $26.4 \pm 8.0^{A}$ & $47.4 \pm 4.5$ & $46.7 \pm 4.0^{B}$ \\
\hline
\end{tabular}

$n=7$ WT-Cre mice, $n=7$ Mtor-cKO mice, $n=8$ 4E-BP1-KO mice, and $n=9$ dKO mice. Measurements are given as mean \pm SD. ${ }^{A} P<0.05$ versus WT-Cre mice; ${ }^{B} P<0.05$ versus 4 weeks after TMX administration Mtor-cKO mice. enhancement of cardiac function as assessed by the dobutamine stress test (Supplemental Figure 5B).

As expected, crossing Mtor-KO mice with 4E-BP1-KO mice completely abolished the accumulation of 4E-BP1 occurring in Mtor-KO mice (Figure 5A). Importantly, dKO mice had significantly better survival than Mtor-cKO mice (Figure 5B). CoxIV was significantly increased in $\mathrm{dKO}$ mice (Figure $5 \mathrm{~A}$ ), suggesting that mitochondrial function was improved. Echocardiographic parameters (Table 3), cell size (Supplemental Figure 2, B and C), and cardiac function (assessed as the LV fractional shortening percentage) (Table 3) were normalized in dKO hearts at 4 weeks after TMX administration, a time at which most Mtor-cKO mice are suffering frank HF. Moreover, the extent of fibrosis was significantly diminished compared with that of Mtor-cKO mice (Supplemental Figure 5, C and D). S6 phosphorylation was increased in $\mathrm{dKO}$ mice, possibly because of the existence of MTOR-independent pathways in regulating S6K activity (Figure 5A). In addition, phosphorylation of Akt was elevated at Thr308 and at Ser473, suggesting that the survival pathways mediated by Akt were activated. This is consistent with a decrease of cleaved Parp protein (Figure 5A) and TUNEL-positive cells (Supplemental Figure 5, E and F) in dKO myocardium. On the other hand, the expression of autophagy genes was similar in Mtor-cKO and dKO mice (data not shown), indicating that autophagy is MTOR-dependent but 4E-BP1-independent and that it is not involved in the amelioration of the HF phenotype in this context.

Expression of constitutively hypophosphorylated $4 E-B P 1$ in cardiomyocytes promotes apoptosis. Because hypophosphorylated 4E-BP1 accumulates in Mtor-cKO myocardium and apoptosis is reduced when MtorcKO mice are crossed with mice lacking Eif4ebp1, we decided to test whether ectopic expression of hypophosphorylated 4E-BP1 induces cell death in cultured cardiomyocytes. To this end, we infected neonatal cardiomyocytes with an adenoviral vector carrying a nonphosphorylatable form of 4E-BP1 (30). We found that this mutant increased the cleavage of caspases 3 and 9 in cardiomyocytes, with respect to infection with an empty vector (Figure 5, C and D). Interestingly, there was no effect on autophagy (Figure 5C and data not shown), in support of the in vivo "rescue" data, demonstrating that autophagy in cardiomyocytes is MTOR dependent but 4E-BP1 independent.

\section{Discussion}

Here, we have assessed the role of cardiac MTOR, showing that Mtor deletion causes a lethal, fully penetrant, dilated-heart phenotype, without passing through an initial hypertrophic phase. Our data support the notion that reduced MTOR signaling is sufficient to induce the development of HF.

Previous reports suggested a key role for decreased MTOR activity in heart disease. Long-term pressure overload was shown to blunt MTOR signaling (4) and reduced MTOR activity was indicated to play a role in the cardiac dysfunction produced by doxorubicin, an anthracycline used as an anticancer agent that can induce dilated cardiomyopathy as a side effect (31). Our results also bear similarities with the effects of Mtor and Raptor deletion in mouse skeletal muscle, where the absence of MTORC1 activity was associated with increased Akt phosphorylation at residues Ser473 and Thr308, progressive muscle degeneration, and premature death $(23,25)$. Interestingly, in Drosophila, which is a simpler model than the mouse, dTOR overactivity and the absence of $d 4 E-B P 1$ were shown to be associated with cardiac malfunction and decreased life span, due to free radical generation $(32,33)$.

In contrast to its role in the development of $\mathrm{HF}$, the role of MTOR in the induction of hypertrophy is better established (2, 34-36). However, whether hypertrophy is beneficial for cardiac function when the heart is under stress remains controversial (27). In mice and rats, pharmacological inhibition of MTOR with rapa- 
mycin was demonstrated to regress remodeling induced by pressure overload $(1,2,37)$ and by myocardial infarction (38), blunting the development of cardiac dysfunction. These studies, while demonstrating that MTOR is critical for the induction of cardiac hypertrophy after stress, suggest that inhibiting this kinase might help abate the worsening of heart function occurring in pathological settings. However, here we have demonstrated that even partial downregulation of MTOR is detrimental when the heart is subjected to pathological stress, because pressure overload accelerated heart dilation and the deterioration of cardiac function of MtorcKO mice expressing reduced, but not null levels, of MTOR. Therefore, adequate MTOR activity and induction of hypertrophy are fundamental for the correct response of the heart to pathological stress. One possible explanation for the discrepancy between our results and those obtained with the use of pharmacological MTOR inhibition might lie in the fact that rapamycin inhibits MTORC2 only with extensive treatment (16), whereas MTOR deletion affects both complexes. Incomplete inhibition of MTOR-dependent survival signaling, via MTORC2-mediated phosphorylation of Akt at Ser473, might then be responsible for the apparent beneficial effects of rapamycin-induced blunting of cardiac hypertrophy. An alternative explanation is that rapamycin may inhibit MTORC1 without altering its stoichiometry, whereas MTOR knockout does alter stoichiometry. On this point, we found rapidly reduced expression of Raptor and increased expression of PRAS40 in MtorcKO myocardium. Free PRAS40 may have effects other than those related to the regulation of MTOR kinase activity and has been discovered to be a direct substrate of MTORC1 itself (39). Further investigation is needed.

A striking feature of Mtor-cKO mice is the accumulation of 4EBP1 within the myocardium. The mechanisms underlying this accumulation are uncertain, but once phosphorylated, 4EB-P1 is ubiquitinated and then degraded (40). Loss of MTOR-mediated phosphorylation of 4E-BP1 could, therefore, be responsible for decreased 4E-BP1 degradation and the buildup of 4E-BP1 in the myocardium. In addition, the finding that deletion of Eif4ebp1 was able to significantly, albeit incompletely, reverse the negative effects produced by the loss of Mtor provides what we believe to be the first evidence for a functional role of 4E-BP1 in HF development. We found that infection of cardiomyocytes with a mutant Eif4ebp1 transgene encoding a nonphosphorylatable molecule induced apoptosis through higher cleaved caspase levels. Similar findings were described in fibroblasts, especially when cultured in the presence of rapamycin $(40,41)$. Thus, these results suggest that, when not inhibited adequately by MTOR, 4E-BP1 is a strong inducer of apoptosis. While the mechanism of 4E-BP1-dependent apoptosis in cardiomyocytes needs further investigation, it has been shown in other cell types that 4E-BPs control the translation efficiency of Gas 2 mRNA, a gene that increases the stability of p53 (42). It is tempting to speculate that this mechanism may also take place in cardiomyocytes. dKO mice might benefit, therefore, from the deletion of Eif4ebp1, because of loss of this death-inducing mechanism. Moreover, because dephosphorylated 4E-BP1 was found in the late (failing) stage of pressure overload in wild-type mice and in the MLP-KO model of HF, it is conceivable that inappropriate inhibitory activity of 4E-BP1, secondary to downregulation of MTOR, might represent a common mechanism in the onset of HF of diverse etiologies.

Inhibition of MTOR can also increase autophagy (10). This is a phenomenon in which a cell digests its own constituents, especially the mitochondria. Active MTOR inhibits the serine/threonine kinase Atg1, a key mediator of autophagy induction (10). Increased cardiomyocyte loss due to autophagy has been reported during progression from compensated hypertrophy to HF and may represent an attempt to remove abnormal proteins or damaged organelles (43). In Mtor-cKO, autophagy and apoptosis are concomitantly increased; however, autophagy is not decreased in the $\mathrm{dKO}$ mice, in contrast to apoptosis, which is dramatically diminished. This suggests that apoptosis is more prominent than autophagy in determining the HF phenotype of Mtor-cKO mice. However, more work is needed to clarify the role of autophagy in our model.

Deletion of Mtor does not immediately affect its 2 complexes identically: MTORC1 activity is more rapidly reduced than that of MTORC2. On this point, phosphatidic acid (PA), a phospholipid required for MTORC assembly, was reported to compete with FKBP12 and rapamycin for interaction with the FKBP12rapamycin-binding (FRB) domain of MTOR, allowing completion of MTORC assembly with either Rictor or Raptor (44). The rate constant of dissociation of MTORC1 from PA and MTOR was suggested to be greater than that of MTORC2 (45). Thus, the higher dissociation rate of MTOR from MTORC1 than that from MTORC2 could explain why MTORC2 is more stable in Mtor-cKO mice.

The results presented in this report on the Mtor-cKO model of HF demonstrate the critical importance of MTOR for appropriate cardiac cell survival and function. As a consequence, the use of chemotherapeutic and immunosuppressive drugs that target MTOR directly or indirectly may very well bear an important risk of aggravating the onset of heart disease in patients with cardiac malfunction. Unfortunately, the effects of MTOR inhibitors on cardiac function in a clinical setting are scarcely described. One study in which renal transplant recipients were switched from a calcineurin-inhibitor immunosuppressive drug to sirolimus (i.e., rapamycin) demonstrated that sirolimus inhibits cardiac hypertrophy associated with kidney transplantation independently of any hemodynamic effect (46). This result, though obtained in a small, nonrandomized clinical trial, uncovers a potentially harmful consequence of sirolimus administration for patients with heart problems. More in-depth studies on cardiac function, before and after high-dose sirolimus administration, are therefore needed. Finally, we believe a novel aspect of this report is that the inhibitory activity of 4E-BP1 is a key mediator of the negative effects of reduced MTOR signaling on cardiomyocyte homeostasis and heart function. Because dephosphorylated 4E-BP1 is increased during latestage $\mathrm{HF}$, it is possible to envision the administration of synthetic molecules that negatively modulate 4E-BP1 activity as a new therapeutic strategy for this syndrome, although the proliferative effects on other tissues must be taken also into account.

\section{Methods}

Gene targeting of MTOR. Genomic MTOR DNA was isolated from R1 ES cells and used to create an Mtor-targeting construct containing floxP sites and the neomycin resistance gene. The construct was generated in the pBluescript II KS+ vector. The $5^{\prime}$ arm of homology consisted of a $3.5-\mathrm{kb}$ Not I-Xho I fragment fused with FRT-Neo-FRT cassette, following the first floxP site upstream of Mtor's 2nd and 3rd exons. The 3' arm of homology was a 3.3-kb Sal I-Bgl II fragment located downstream of a second floxP site (Supplemental Figure 1A). The targeting construct was verified by sequencing and linearized with Bgl II, before electroporation into R1 ES cells at the Transgenic Core Facility at the University of California San Diego. 
Southern blot analysis. Genomic DNA was extracted from G418-resistant ES cell clones and mouse tails as previously described (47). ES cell DNA was digested with Hind I and analyzed by Southern blot analysis. A 315-bp fragment was generated by PCR using genomic mouse DNA and specific Mtor primers (forward, TTGTGCTGAGATATGGAAGCAGGGTGAGGAGAAC; reverse, CTCCGCTCTTCCCGGCTACTCTAGCCTCCC). The PCR product was subsequently radiolabeled, using $\alpha-\left[{ }^{32} \mathrm{P}\right] \mathrm{dCTP}$, by random priming (Invitrogen). DNA blots were hybridized with the radiolabeled probe and visualized by autoradiography. The wild-type allele is represented by a $12.5-\mathrm{kb}$ band, whereas a $9-\mathrm{kb}$ band represents the correctly targeted mutant allele (Supplemental Figure 1B). Four clones out of six hundred G418-resistant ES clones that had undergone homologous recombination were identified by Southern blot analysis.

Generation and genotyping of Mtor knockout mice and Mtor-cKO/4E-BP1 $d K O$ mice. Two independent homologous recombinant ES clones were microinjected into blastocysts from C57BL/6 mice at the Transgenic Core Facility at the University of California San Diego. Male chimeras were inbred with female $\mathrm{C} 57 \mathrm{BL} / 6$ mice to generate germ line-transmitted heterozygous mice (Mtorfl/+ mice). To delete the Neo cassette, heterozygous Flox-FRT mice were crossed with Flpe deleter mice from The Jackson Laboratories (48). After that, $M$ tor $^{f /+}$ mice were backcrossed to $\mathrm{C} 57 \mathrm{BL} / 6$ for more than 8 generations (F8 offspring) and subsequently intercrossed to generate homozygous null mutant mice (Mtor flf $/ f)^{2}$. Offspring were genotyped by PCR analysis using mouse tail DNA and wild-type (forward, GCTCACTGTACTCTGTCTGCACTTG; reverse, GAAATAGCACGCATTTCTACTTG) and knockout allele-specific primers (forward, GTACACTCTCCTTAAAGTAGCTTCCC; reverse primer was the same as that used for wild type) (Supplemental Figure 1C). Cardiac-specific MTOR-inducible KO mice ( $\alpha$-MHC-MerCreMer/Mtorf/fl) were generated by breeding $M t o f^{f / f l}$ mice with mice expressing the cardiac-specific MerCreMer $\alpha$-MHC promoter-driven cre recombinase gene (provided by J.D. Molkentin, University of Cincinnati, Cincinnati, Ohio, USA) (49). Two-month-old male mice were administered TMX (T5648, Sigma-Aldrich), dissolved in sesame oil at $30 \mathrm{mg} / \mathrm{kg} / \mathrm{d}$, with i.p. injection for 6 days, a dose that does not produce toxicity in mice, in order to induce the knockout of Mtor (Supplemental Figure 1D). Control animals used in this study were WT male mice carrying only one copy of the aMHC-MCM transgene (WT-Cre mice) and Mtor ${ }^{f / / f}$ mice not expressing the cre recombinase gene that were treated with the same TMX regimen (F/F mice) as well as $\alpha$ MHC-MCM/Mtor ${ }^{f / f l}$ mice not receiving TMX.

Cardiac-specific MTOR-inducible KO mice were also crossbred with mice deficient for Eif4ebp1 (29) to generate double-knockout mice (MtorcKO/4E-BP1-KO mice). The resulting background strains were C57BL/6 and were unchanged throughout the study.

All animal procedures were carried out in accordance with the guidelines set by the University of California San Diego Institutional Animal Care Program and with IACUC approval.

Protein analysis and antibodies. Western blot analyses were performed to evaluate heart protein levels/phosphorylation states after fractionation, following standard procedures. Antibodies used in this study were purchased from Cell Signaling Technology, Santa Cruz Biotechnology Inc., Sigma-Aldrich, and Millipore. ImageJ software $(\mathrm{NIH})$ was used to perform densitometric analyses (http://rsb.info.nih.gov/ij/).

Histochemistry and analysis of cell size on myocardial tissue. Standard techniques were adopted as previously described (50). In brief, hearts were flushed with PBS, relaxed in $50 \mathrm{mM} \mathrm{KCl}$ in PBS, and fixed in $10 \%$ neutralbuffered formalin (Sigma-Aldrich) overnight. The cardiomyocyte crosssectional area was measured from $8-\mu \mathrm{m}$-thick heart sections that had been stained with FITC-conjugated wheat germ agglutinin, using OpenLab software (Improvision). Only myocytes that were round (i.e., were cut in cross section) were included in the analysis. An observer blind to the genotypes of the heart samples performed the measurements.

Transmission electron microscopy. Hearts were retrogradely perfused with 50 $\mathrm{mM} \mathrm{KCl}$ in tyrode solution, followed with $2 \%$ paraformaldehyde $/ 2 \%$ glutaraldehyde in $150 \mathrm{mM}$ sodium cacodylate buffer. The LV tissues were processed for transmission electron microscopy as described previously (51).

Cultured adult mouse cardiomyocytes. Adult ventricular myocytes were isolated as described previously (52) and plated at a density of about 5,000 myocytes $/ \mathrm{cm}^{2}$ on dishes precoated with laminin in MEM supplemented with $1 \mathrm{mM} \mathrm{CaCl}_{2}, 15 \mathrm{mM}$ butanedione monoxime, $25 \mathrm{mM}$ HEPES, and penicillin plus streptomycin $1 \%$.

$\left[{ }^{3} H\right]$ lencine uptake assay. Adult cardiomyocytes were used to assess incorporation of $\left[{ }^{3} \mathrm{H}\right]$ leucine. Serum-deprived (16 hours) myocytes were incubated in culture medium containing $1 \mu \mathrm{Ci} / \mathrm{ml}\left[{ }^{3} \mathrm{H}\right]$ leucine (Amersham and PerkinElmer) in the presence of fetal bovine serum for 20 hours before assaying to measure radioactivity (52).

Infection of neonatal cardiomyocytes with adenovirus. One-day-old neonatal mouse cardiomyocytes (nCMCs) were used to assess apoptotic effects of overexpression of hypophosphorylated 4E-BP1. The culture medium was switched to serum-free medium 24 hours before adenovirus infection. nCMCs were mock and Adeno-4EBP1-5A infected with serial MOIs (2, 10, 20 , and $50 \mathrm{MOI}$ ) for 24 hours and 72 hours before Western blotting or immunostaining. Mock infection was performed with an empty adenoviral vector. Adeno-4E-BP1-5A (a gift from S. Huang, Health Sciences Center, Louisiana State University, Shreveport, Louisiana, USA) encodes a constitutively hypophosphorylated, mutated (T37A, T46A, S65A, T70A, and S83A) 4E-BP1 and therefore has a dominant-positive function. Cultured nCMCs were fixed with paraformaldehyde, permeabilized, and stained with anti-cleaved caspase $3 / 9$ and anti- $\alpha$-actinin antibodies.

Morphometric measurement of isolated cardiomyocytes. To measure cardiomyocyte size in formalin-fixed adult hearts, hearts (with atria removed) were digested with $50 \% \mathrm{KOH}$ for 24 hours $(53,54)$. After careful washing with PBS, typical rod-shaped cardiomyocytes only were analyzed for the morphometric measurements. Using NIH ImageJ software, long axis, short axis, and cell area were measured and compared. An average of 300-400 cells were chosen at random for measurements.

Pressure overload cardiac hypertrophy. We used 2-month-old male mice. Pressure overload was obtained through transverse aortic arch constriction under anesthesia, as described previously (55).

Echocardiography assessment of cardiac function. Mice were anesthetized with isoflurane and subjected to echocardiography as previously described (50). The chest was shaved, and the mouse laid supine. A Philips SONOS 5500, equipped with a $15-\mathrm{MHz}$ linear array transducer, was used for noninvasive transthoracic echocardiography. Two-dimensional guided M-mode tracings were recorded. The internal diameter of the LV in the short-axis plane was measured at end diastole and end systole from M-mode recordings just below the tips of the mitral valve leaflets. The interventricular and LV posterior wall thicknesses were measured at end diastole. Heart contractility, assessed as the LV fractional shortening percentage, was calculated as described previously (56). Echocardiography was performed by an observer who obtained all images and measurements without knowledge of the genotypes of the animals.

Hemodynamic assessment of cardiac function. Mice were anesthetized by i.p. injection with ketamine $(100 \mathrm{mg} / \mathrm{kg})$ and xylazine $(5.0 \mathrm{mg} / \mathrm{kg})$. After intubation, mice were ventilated, and the carotid artery was cannulated for the measurement of arterial pressure. A high fidelity 1.4F micromanometer catheter (Millar Instruments) was inserted into the LV through the right carotid artery and secured in position. The femoral vein was cannulated and used for infusion of dobutamine. After bilateral vagotomy, continuous high fidelity LV pressure was recorded at baseline and about 3 minutes 
after each dose of dobutamine. The parameters measured were heart rate, aortic pressure, LV systolic and diastolic pressure, and the maximal and minimal first derivative of $\mathrm{LV}$ pressure $(\mathrm{dP} / \mathrm{dtMax}$ and $\mathrm{dP} / \mathrm{dtMin}$, respectively). Twelve sequential beats were averaged for each measurement (50).

TUNEL staining for apoptotic-related proteins. TUNEL staining was done as previously described (50). The In Situ Cell Death Detection Kit (Clonetech) was used for TUNEL; samples were processed according to the manufacturer's recommendations. Sections were mounted on coverslips and evaluated by fluorescence microscopy. All the TUNEL-positive cells in an entire section were counted. To determine the number of nuclei in a section, 4 optical fields were counted, and the average number of nuclei per unit area was multiplied by the total area of the section, as measured in a dark-field image.

Quantitative real-time PCR for fetal genes and Eif4ebp1. Total RNA was extracted from LV myocardium using TRIzoL reagent (Invitrogen), according to the manufacturer's protocol. One microgram of total RNA from each specimen was reverse transcribed to cDNA using SuperScript III Reverse Transcriptase and random hexamers as primers (Invitrogen). Quantitative real-time PCR (qRT-PCR) was performed with an ABI PRISM 7300 instrument (Applied Biosystems) using TaqMan probes (Assay on Demand; Applied Biosystems) for cardiac fetal genes (Nppa, Mm01255748_g1; Myb7, Mm00600555_m1; and Acta1, Mm00808218_g1) and SYBR Green (Applied Biosystems) for the Eif4ebp1 transcript (forward, 5'-GAGAGCTGCACAGCATTCA G-3'; reverse, $5^{\prime}$-AGTCATTCCCCTGCAGTAGC-3'). Gapdh was used as internal control. $\Delta \Delta^{\mathrm{CT}}$ values were calculated as previously described $(57,58)$.

eIF4E pull-down $m^{7}$ GTP-sepharose chromatography. Briefly, $20 \mu \mathrm{l}$ of a $50 \%$ slurry of $\mathrm{m}^{7}$ GTP-Sepharose CL-4B (GE Healthcare, Amersham Biosciences) was rotated with $1 \mathrm{mg}$ heart lysate for 1 hour at $4^{\circ} \mathrm{C}$. The $\mathrm{m}^{7} \mathrm{GTP}$-Sepharose was pelleted by centrifugation and washed twice in lysis buffer. For
SDS-PAGE, proteins bound to $\mathrm{m}^{7} \mathrm{GTP}$-Sepharose were released by boiling them in the sample buffer and processed for SDS-PAGE and immunoblotting as previously described (59).

Statistics. Statistical comparison was performed in at least 3 independent experiments using paired or unpaired 2-tailed Student's $t$ test, whereas comparison between groups was analyzed by 1 -way repeated-measures ANOVA, combined with a Newman-Keuls post-test to compare different values using Prism 4.0 software (GraphPad Software Inc.). $P$ values of less than 0.05 were considered statistically significant.

\section{Acknowledgments}

We thank Jeffrey D. Molkentin for $\alpha$-MHC-MerCreMer mice, Shile Huang for Adeno-HA-4E-BP1-5A construct, and Estela Jacinto and Bing Su for Raptor, Rictor, and $\mathrm{mSin} 1$ antibodies. This work was sponsored by grants from the NIH (HL078797-01A1 to G. Condorelli, J. Chen, and J.H. Brown), the Perlman Fund for Cardiovascular Research and Education and the San Diego Foundation for Cardiovascular Research and Education (to K.L. Peterson), the CARIPLO Foundation (2008.2504 to D. Catalucci), and the Canadian Institutes for Health Research and the Howard Hughes Medical Institute (to N. Sonenberg).

Received for publication March 16, 2010, and accepted in revised form June 9, 2010.

Address correspondence to: Gianluigi Condorelli, Department of Medicine, University of California San Diego, 9500 Gilman Drive, La Jolla, California 92093-0613, USA. Phone: 858.822.5563; Fax: 858.822.3027; E-mail: gcondorelli@ucsd.edu.
1. McMullen JR, et al. Inhibition of mTOR signaling with rapamycin regresses established cardiac hypertrophy induced by pressure overload. Circulation. 2004;109(24):3050-3055

2. Shioi $T$, et al. Rapamycin attenuates load-induced cardiac hypertrophy in mice. Circulation. 2003; 107(12):1664-1670.

3. Hill JA, Olson EN. Cardiac plasticity. $N$ Engl J Med. 2008;358(13):1370-1380.

4. Kemi OJ, et al. Activation or inactivation of cardiac Akt/mTOR signaling diverges physiological from pathological hypertrophy. J Cell Physiol. 2008; 214(2):316-321.

5. Dunlop EA, Tee AR. Mammalian target of rapamycin complex 1: signalling inputs, substrates and feedback mechanisms. Cell Signal. 2009;21(6):827-835.

6. Wullschleger S, Loewith R, Hall MN. TOR signaling in growth and metabolism. Cell. 2006;124(3):471-484.

7. Gingras AC, et al. Regulation of 4E-BP1 phosphorylation: a novel two-step mechanism. Genes Dev. 1999;13(11):1422-1437.

8. Kantidakis T, Ramsbottom BA, Birch JL, Dowding SN, White RJ. mTOR associates with TFIIIC, is found at tRNA and $5 S$ rRNA genes, and targets their repressor Mafl. Proc Natl Acad Sci U S A. 2010; 107(26):11823-11828.

9. Cunningham JT, Rodgers JT, Arlow DH, Vazquez F, Mootha VK, Puigserver P. mTOR controls mitochondrial oxidative function through a YY1PGC-1 $\alpha$ transcriptional complex. Nature. 2007; 450(7170):736-740.

10. Chan EY. mTORC1 phosphorylates the ULK1mAtg13-FIP200 autophagy regulatory complex. Sci Signal. 2009;2(84):pe51.

11. Cybulski N, Hall MN. TOR complex 2: a signaling pathway of its own. Trends Biochem Sci. 2009; 34(12):620-627.

12. Ikenoue $\mathrm{T}$, Inoki $\mathrm{K}$, Yang $\mathrm{Q}$, Zhou $\mathrm{X}$, Guan KL. Essential function of TORC2 in PKC and Akt turn motif phosphorylation, maturation and signalling. EMBO J. 2008;27(14):1919-1931.

13. Facchinetti $\mathrm{V}$, et al. The mammalian target of rapamycin complex 2 controls folding and stability of Akt and protein kinase C. EMBO J. 2008; 27(14):1932-1943.

14. Garcia-Martinez JM, Alessi DR. mTOR complex 2 (mTORC2) controls hydrophobic motif phosphorylation and activation of serum- and glucocorticoidinduced protein kinase 1 (SGK1). Biochem J. 2008; 416(3):375-385.

15. Sarbassov DD, Guertin DA, Ali SM, Sabatini DM. Phosphorylation and regulation of Akt/ PKB by the rictor-mTOR complex. Science. 2005; 307(5712):1098-1101.

16. Sarbassov DD, et al. Prolonged rapamycin treatment inhibits mTORC2 assembly and Akt/PKB. Mol Cell. 2006;22(2):159-168.

17. Ceci M, et al. Cardiac-specific overexpression of E40K active Akt prevents pressure overload-induced heart failure in mice by increasing angiogenesis and reducing apoptosis. Cell Death Differ. 2007; 14(5):1060-1062.

18. DeBosch B, et al. Akt1 is required for physiological cardiac growth. Circulation. 2006;113(17):2097-2104.

19. GangloffYG, et al. Disruption of the mouse mTOR gene leads to early postimplantation lethality and prohibits embryonic stem cell development. $\mathrm{Mol}$ Cell Biol. 2004;24(21):9508-9516.

20. Murakami M, et al. mTOR is essential for growth and proliferation in early mouse embryos and embryonic stem cells. Mol Cell Biol. 2004;24(15):6710-6718.

21. Guertin DA, et al. Ablation in mice of the mTORC components raptor, rictor, or mLST8 reveals that mTORC2 is required for signaling to Akt-FOXO and PKC $\alpha$, but not S6K1. Dev Cell. 2006;11(6):859-871.

22. Crone SA, et al. ErbB2 is essential in the prevention of dilated cardiomyopathy. Nat Med. 2002;8(5):459-465.

23. Bentzinger CF, et al. Skeletal muscle-specific abla- tion of raptor, but not of rictor, causes metabolic changes and results in muscle dystrophy. Cell Metab. 2008;8(5):411-424.

24. Um SH, et al. Absence of S6K1 protects against ageand diet-induced obesity while enhancing insulin sensitivity. Nature. 2004;431(7005):200-205.

25. Risson V, et al. Muscle inactivation of mTOR causes metabolic and dystrophin defects leading to severe myopathy. J Cell Biol. 2009;187(6):859-874.

26. Arber S, et al. MLP-deficient mice exhibit a disruption of cardiac cytoarchitectural organization, dilated cardiomyopathy, and heart failure. Cell. 1997; 88(3):393-403.

27. Frey N, Katus HA, Olson EN, Hill JA. Hypertrophy of the heart: a new therapeutic target? Circulation. 2004;109(13):1580-1589.

28. McMullen JR, et al. Deletion of ribosomal S6 kinases does not attenuate pathological, physiological, or insulin-like growth factor 1 receptorphosphoinositide 3-kinase-induced cardiac hypertrophy. Mol Cell Biol. 2004;24(14):6231-6240.

29. Tsukiyama-Kohara K, et al. Adipose tissue reduction in mice lacking the translational inhibitor 4EBP1. Nat Med. 2001;7(10):1128-1132.

30. Mothe-Satney I, Yang D, Fadden P, Haystead TA, Lawrence JC Jr. Multiple mechanisms control phosphorylation of PHAS-I in five (S/T)P sites that govern translational repression. Mol Cell Biol. 2000; 20(10):3558-3567.

31. $\mathrm{Zhu} \mathrm{W}$, et al. Acute doxorubicin cardiotoxicity is associated with p53-induced inhibition of the mammalian target of rapamycin pathway. Circulation. 2009;119(1):99-106.

32. Lee JH, et al. Sestrin as a feedback inhibitor of TOR that prevents age-related pathologies. Science. 2010; 327(5970):1223-1228.

33. Topisirovic I, Sonenberg N. Cell biology. Burn out or fade away? Science. 2010;327(5970):1210-1211.

34. Shen WH, et al. Cardiac restricted overexpression 
of kinase-dead mammalian target of rapamycin (mTOR) mutant impairs the mTOR-mediated signaling and cardiac function. J Biol Chem. 2008; 283(20):13842-13849.

35. Boluyt MO, et al. Rapamycin inhibits alpha $1-$ adrenergic receptor-stimulated cardiac myocyte hypertrophy but not activation of hypertrophyassociated genes. Evidence for involvement of p70 S6 kinase. Circ Res. 1997;81(2):176-186.

36. Ikeda Y, et al. Cardiac-specific deletion of LKB1 leads to hypertrophy and dysfunction. J Biol Chem. 2009;284(51):35839-35849.

37. Gao XM, et al. Inhibition of mTOR reduces chronic pressure-overload cardiac hypertrophy and fibrosis. J Hypertens. 2006;24(8):1663-1670.

38. Buss SJ, et al. Beneficial effects of Mammalian target of rapamycin inhibition on left ventricular remodeling after myocardial infarction. J Am Coll Cardiol. 2009;54(25):2435-2446.

39. Fonseca BD, Smith EM, Lee VH, MacKintosh C, Proud CG. PRAS40 is a target for mammalian target of rapamycin complex 1 and is required for signaling downstream of this complex. J Biol Chem. 2007; 282(34):24514-24524.

40. Elia A, Constantinou C, Clemens MJ. Effects of protein phosphorylation on ubiquitination and stability of the translational inhibitor protein $4 \mathrm{E}$ BP1. Oncogene. 2008;27(6):811-822.

41. Li S, et al. Translational control of cell fate: availability of phosphorylation sites on translational repressor 4E-BP1 governs its proapoptotic potency. Mol Cell Biol. 2002;22(8):2853-2861.
42. Sonenberg N, Hinnebusch AG. Regulation of translation initiation in eukaryotes: mechanisms and biological targets. Cell. 2009;136(4):731-745.

43. Nishida K, Kyoi S, Yamaguchi O, Sadoshima J, Otsu K. The role of autophagy in the heart. Cell Death Differ. 2009;16(1):31-38.

44. Foster DA, Toschi A. Targeting mTOR with rapamycin: one dose does not fit all. Cell Cycle. 2009; 8(7):1026-1029.

45. Toschi A, Lee E, Xu L, Garcia A, Gadir N, Foster DA Regulation of mTORC1 and mTORC2 complex assembly by phosphatidic acid: competition with rapamycin. Mol Cell Biol. 2009;29(6):1411-1420.

46. Paoletti E, Amidone M, Cassottana P, Gherzi M, Marsano L, Cannella G. Effect of sirolimus on left ventricular hypertrophy in kidney transplant recipients: a 1-year nonrandomized controlled trial. Am J Kidney Dis. 2008;52(2):324-330.

47. Chen J, et al. Selective requirement of myosin light chain $2 \mathrm{v}$ in embryonic heart function. J Biol Chem. 1998;273(2):1252-1256.

48. Kanki H, Suzuki H, Itohara S. High-efficiency CAG-FLPe deleter mice in C57BL/6J background. Exp Anim. 2006;55(2):137-141.

49. Sohal DS, et al. Temporally regulated and tissue-specific gene manipulations in the adult and embryonic heart using a tamoxifen-inducible Cre protein. Circ Res. 2001;89(1):20-25.

50. Condorelli $\mathrm{G}$, et al. Akt induces enhanced myocardial contractility and cell size in vivo in transgenic mice. Proc Natl Acad Sci U S A. 2002;99(19):12333-12338.

51. Claudio PP, et al. Adenoviral RB2/p130 gene trans- fer inhibits smooth muscle cell proliferation and prevents restenosis after angioplasty. Circ Res. 1999; 85(11):1032-1039.

52. Catalucci D, et al. Akt regulates L-type $\mathrm{Ca} 2+$ channel activity by modulating Cavalpha1 protein stability. J Cell Biol. 2009;184(6):923-933.

53. Gerdes AM, et al. New method to evaluate myocyte remodeling from formalin-fixed biopsy and autopsy material. J Card Fail. 1998;4(4):343-348.

54. Engel D, Peshock R, Armstong RC, Sivasubramanian N, Mann DL. Cardiac myocyte apoptosis provokes adverse cardiac remodeling in transgenic mice with targeted TNF overexpression. Am J Physiol Heart Circ Physiol. 2004;287(3):H1303-H1311.

55. Rockman HA, et al. Segregation of atrial-specific and inducible expression of an atrial natriuretic factor transgene in an in vivo murine model of cardiac hypertrophy. Proc Natl Acad Sci U S A. 1991; 88(18):8277-8281.

56. Tanaka N, et al. Transthoracic echocardiography in models of cardiac disease in the mouse. Circulation. 1996;94(5):1109-1117.

57. Care A, et al. MicroRNA-133 controls cardiac hypertrophy. Nat Med. 2007;13(5):613-618.

58. Elia L, et al. The knockout of miR-143 and -145 alters smooth muscle cell maintenance and vascular homeostasis in mice: correlates with human disease. Cell Death Differ. 2009;16(12):1590-1598.

59. Wang L, Proud CG. Ras/Erk signaling is essential for activation of protein synthesis by Gq proteincoupled receptor agonists in adult cardiomyocytes. Circ Res. 2002;91(9):821-829. 